\title{
Hipoglicemia no Diabetes Autoimune Latente do Adulto (LADA) associada ao distúrbio hipocalêmico: relato de caso
}

\author{
Hypoglycemia in Adult Latent Autoimmune Diabetes (LADA) associated with hypokalemic \\ disorder: case report \\ Hipoglucemia en adultos con Diabetes Autoinmune Latente (LADA) asociada con transtorno \\ hipopotasémico: reporte de caso
}

\author{
Bárbara Queiroz de Figueiredo \\ ORCID: https://orcid.org/0000-0003-1630-4597 \\ Centro Universitário de Patos de Minas, Brasil \\ E-mail: barbarafigueiredo@unipam.edu.br \\ Dalbert Samuel Dutra \\ ORCID: https://orcid.org/0000-0001-8417-1138 \\ Centro Universitário de Patos de Minas, Brasil \\ E-mail: dalbertdutra@gmail.com \\ Izabella Araújo de Oliveira \\ ORCID: https://orcid.org/0000-0001-5105-463X \\ Centro Universitário de Patos de Minas, Brasil \\ E-mail: araujoo.izabella@gmail.com \\ Laila Regina Pereira Lopes \\ ORCID: https://orcid.org/0000-0002-5004-7873 \\ Centro Universitário de Patos de Minas, Brasil \\ E-mail: lailalopes@unipam.edu.br \\ Murilo Henrique Godinho Roque \\ ORCID: https://orcid.org/0000-0001-9043-7043 \\ Centro Universitário de Patos de Minas, Brasil \\ E-mail: murilogodinho@unipam.edu.br \\ Mychell Flávio Aparecido Rodrigues de Sousa \\ ORCID: https://orcid.org/0000-0003-1132-2761 \\ Centro Universitário de Patos de Minas, Brasil \\ E-mail: mychel.flavio@hotmail.com \\ Vanessa Pereira Tolentino \\ ORCID: https://orcid.org/0000-0003-2566-2222 \\ Centro Universitário de Patos de Minas, Brasil \\ E-mail: vanessapt@unipam.edu.br
}

\begin{abstract}
Resumo
Introdução: O diabetes latente do adulto (LADA), constitui-se como um tipo de diabetes mellitus do tipo 1, em que a principal causa é a falência primária na produção pancreática de insulina, de maneira autoimune. Metodologia: Tratase de relato de caso clínico de análise descritiva com aporte de trabalhos acadêmicos entre os anos de 2001 e 2020. Descrição do caso: Paciente do sexo feminino, 65 anos de idade, diagnosticada com LADA, faz uso diário de insulinas de ação intermediária e lenta, bem como de medicamentos anti-diabéticos orais, deu entrada no hospital com quadro de hipoglicemia severa (glicemia capilar de $51 \mathrm{mg} / \mathrm{dl}$ ), rebaixamento do estado de consciência, taquicardia, fraqueza e espasmos musculares, bem como sensação de câimbra nos membros inferiores. Exames laboratoriais mostraram, por meio do método de eletrodo seletivo, que os níveis séricos de potássio estavam em 2,80 mEq/L (VR: 3,50 e 5,50 mEq/L), constituindo-se como uma hipocalemia. Discussão: Haja vista que a insulina é capaz de mobilizar o potássio para dentro da célula, pequenas alterações no potássio sérico podem implicar profunda alteração na condução e excitabilidade do coração, podendo alterar a função e o ritmo cardíacos e, inclusive, causar parada cardiorrespiratória. Conclusão: Sob episódios de hipoglicemias severas, que podem ser corroboradas pelo uso indiscriminado de doses insulínicas, uma das exacerbações do episódio é o desequilíbrio eletrolítico, em suma, o potássio, haja vista o poder da insulina de realocar esse eletrólito para o meio intracelular, diminuindo, assim, os níveis séricos e exacerbando-se com a hipocalemia.
\end{abstract}

Palavras-chave: Hipocalemia; Diabetes Melitos; LADA; Hipoglicemia. 


\begin{abstract}
Introduction: Adult latent diabetes (LADA) is a type 1 diabetes mellitus, in which the main cause is the primary failure in the pancreatic insulin production, in an autoimmune way. Methodology: This is a clinical case report of descriptive analysis with contribution of academic works between the years 2001 and 2020. Case description: Female patient, 65 years old, diagnosed with LADA, makes daily use of insulin from intermediate and slow action, as well as oral anti-diabetic drugs, he was admitted to the hospital with severe hypoglycemia (capillary blood glucose of $51 \mathrm{mg} / \mathrm{dl}$ ), reduced state of consciousness, tachycardia, weakness and muscle spasms, as well as a feeling of cramp in the lower limbs. Laboratory tests showed, through the selective electrode method, that serum potassium levels were $2.80 \mathrm{mEq} / \mathrm{L}$ (VR: 3.50 and $5.50 \mathrm{mEq} / \mathrm{L}$ ), constituting hypokalemia. Discussion: Given that insulin is capable of mobilizing potassium into the cell, small changes in serum potassium may imply profound changes in the conduction and excitability of the heart, which may alter cardiac function and rhythm and even cause cardiorespiratory arrest. Conclusion: Under episodes of severe hypoglycemia, which can be corroborated by the indiscriminate use of insulin doses, one of the exacerbations of the episode is electrolyte imbalance, in short, potassium, given the power of insulin to relocate this electrolyte to the intracellular environment, thus decreasing serum levels and exacerbating with hypokalemia.
\end{abstract}

Keywords: Hypokalemia; Diabetes Mellitus; LADA; Hypoglycemia.

\title{
Resumen
}

Introducción: la diabetes latente del adulto (LADA) es una diabetes mellitus tipo 1, en la que la principal causa es el fallo primario en la producción de insulina pancreática, de forma autoinmune. Metodología: Se trata de un reporte de caso clínico de análisis descriptivo con aporte de trabajos académicos entre los años 2001 y 2020. Descripción del caso: Paciente de sexo femenino, 65 años, diagnosticada de LADA, hace uso diario de insulina de acción intermedia y lenta, así como antidiabético oral, ingresó en el hospital con hipoglucemia severa (glucemia capilar de $51 \mathrm{mg} / \mathrm{dl}$ ), estado de conciencia reducido, taquicardia, debilidad y espasmos musculares, así como sensación de calambre en miembros inferiores. Las pruebas de laboratorio mostraron, mediante el método de electrodo selectivo, que los niveles de potasio sérico eran de 2,80 mEq / L (VR: 3,50 y 5,50 mEq / L), constituyendo hipopotasemia. Discusión: Dado que la insulina es capaz de movilizar potasio hacia la célula, pequeños cambios en el potasio sérico pueden implicar cambios profundos en la conducción y excitabilidad del corazón, que pueden alterar la función y el ritmo cardíaco e incluso provocar un paro cardiorrespiratorio. Conclusión: En episodios de hipoglucemia severa, que pueden ser corroborados por el uso indiscriminado de dosis de insulina, una de las exacerbaciones del episodio es el desequilibrio electrolítico, en definitiva, el potasio, dado el poder de la insulina para reubicar este electrolito al medio intracelular. disminuyendo así los niveles séricos y exacerbando con hipopotasemia.

Palabras clave: Hipopotassemia; Diabetes Mellitus; LADA; Hipoglucemia.

\section{Introdução}

O diabetes mellitus (DM) é uma condição crônica de saúde muito prevalente em todos os países. A Organização Mundial de Saúde (OMS) estima que mais de 500 milhões de pessoas vivem, atualmente com DM em todo o mundo. A classificação atual do DM é baseada nos mecanismos fisiopatológicos que levam à hiperglicemia. No DM do tipo 1 (DM1) ocorre deficiência total ou quase total da produção de insulina pelas células beta das ilhotas pancreáticas enquanto no DM do tipo 2 (DM2) o que predomina é a resistência à ação da insulina nos tecidos associada a graus variáveis de deficiência da produção pancreática da mesma (Sociedade Brasileira de Diabetes, 2020).

O conceito de diabetes autoimune latente do adulto, cuja sigla em inglês é LADA, de latent autoimmune diabetes in adults foi introduzido pela primeira vez em 1993, por Tuomi e Zimmet para definir pacientes diabéticos adultos que não requeriam insulina para controle da hiperglicemia nas fases iniciais da patologia, mas apresentavam autoanticorpos contra proteínas das células-beta e progressão mais rápida para necessidade de insulina. Trata-se de uma forma de diabetes em que a autoimunidade contra as células beta pancreáticas se desenvolve de maneira mais arrastada e a obrigatoriedade do uso da insulina pode se dar após várias teorias diagnósticas. No LADA, a disfunção da célula $\beta$ tem sido reportada como intermediária entre os dois principais tipos de diabetes (DM1 e DM2) (Calsolari et al. 2007).

O LADA, por definição, acomete pessoas de 25 anos ou mais, mas pode incidir em qualquer idade fazendo com que, muitas vezes, o paciente seja diagnosticado como tendo DM2. Do ponto de vista fisiopatológico, o DM1 resulta da falta absoluta ou quase absoluta de insulina enquanto o DM2 ocorre por resistência à ação da insulina nos tecidos e graus variáveis de redução na produção do hormônio. Fenômenos genéticos, imunológicos e metabólicos parecem convergir com outros 
processos de doença condicionando a apoptose da célula $\beta$ e contribuindo para o aparecimento da LADA. De acordo com Jones et al. (2021), sendo ela identificada com um grupo de características clínicas e genéticas intermediárias entre os dois tipos de diabetes, nota-se que esse fenômeno ainda não está claro, sendo propostas, pelo autor, três teorias: como um fenótipo mais brando que inicia-se em idades mais tardias e que são semelhantes ao DM2, mas que requer insulina; como uma síndrome em que as fisiopatologias do DM1 e DM2 estão presentes em cada paciente; como um grupo heterogêneo decorrente de dificuldades de classificação.

O diagnóstico de LADA é tipicamente baseado no achado de hiperglicemia, associado à constatação de que a causa seja devida à uma falha das ilhotas de Langherans em produzir insulina, presença de sintomas agudos, índice de massa corporal $<25 \mathrm{~kg} / \mathrm{m} 2$, história pessoal ou familiar de outras doenças autoimunes. A presença de, pelo menos, duas destas características clínicas justifica o pedido de anticorpos contra as células beta pancreáticas. Tem-se, atualmente, quatro tipos de anticorpos: anti-glutamato descarboxilase (GADA), anti-insulina (IAA), anti-células beta (ICA) e antitransportador de zinco. No LADA, os anticorpos anti-insulina (IAA) são raros, enquanto os GADA são característicos, mas estes podem se tornar negativos ao longo do tempo. Indivíduos com título elevado de GADA ou com positividade para maior número de anticorpos, geralmente apresentam um índice de massa corporal (IMC) inferior, menor secreção endógena de insulina e progressão mais rápida para insulinoterapia. Assim, o título de GADA pode permitir identificar indivíduos que respondem pior à terapêutica com antidiabéticos orais e que apresentam maior risco de cetoacidose (Silva, 2007).

A detecção de um nível baixo de peptídeo-C e anticorpos contra as ilhotas de Langerhans reforçam e sustentam este diagnóstico. Uma diferenciação com o diabetes tipo 2 pode ser feita porque as pessoas com LADA tipicamente têm níveis baixos, embora às vezes moderados, de peptídeo $\mathrm{C}$ à medida que a doença progride, e aqueles com resistência à insulina ou diabetes tipo 2 são mais propensos a ter níveis elevados de peptídeo $\mathrm{C}$ devido a uma produção excessiva de insulina. Clinicamente, os indivíduos com LADA representam um grupo heterogêneo de doentes com títulos variáveis de anticorpos, índice de massa corporal (IMC) e progressão para insulinoterapia (Couri et al. 2009).

Com base no consenso da Associação Americana de Diabetes (ADA) e da Associação Europeia para o Estudo do Diabetes (EASD) de 2020 para a DM2 e a heterogeneidade dentro do diabetes autoimune latente do adulto, propôs-se "desvios" para o LADA dessas diretrizes. Três grandes categorias de níveis aleatórios de peptídeo C foram introduzidas pelo painel: níveis de peptídeo $\mathrm{C}<0,3 \mathrm{nmol} / \mathrm{L}$ como um regime de insulina múltipla recomendado como para tratamento como DM1; Valores de peptídeo $\mathrm{C} \geq 0,3$ e $\leq 0,7 \mathrm{nmol}$ / L: definido pelo painel como uma "área cinza" na qual um algoritmo ADA / EASD modificado para tratamento como DM2 é recomendado, considerando a insulina em combinação com outras terapias para modular a insuficiência das células $\beta$ e limitar as complicações diabéticas; Valores de peptídeo C> 0,7 nmol / L: sugestivo de um algoritmo ADA / EASD modificado como para tratamento como DM2, mas permitindo a natureza potencialmente progressiva de LADA monitorando o peptídeo C para ajustar o tratamento (Buzzeti et al. 2020).

Sabe-se que independentemente da idade, a insulinoterapia deve ser iniciada em pacientes que apresentem hiperglicemia acima de $280 \mathrm{mg} / \mathrm{dL}$, acompanhada de sintomas como poliuria, polidipsia, perda de peso e astenia. Muitas vezes, com a normalização da glicemia, a glicotoxicidade desaparece, sendo que, nesses casos, é possível a redução da dose ou tentativa de manter a glicemia por meio de medicações orais. Além disso, a monitorização domiciliar deve ser constante, principalmente em idosos, haja vista que os eventos hipoglicêmicos, por exemplo, tendem a ser mais frequentes e deletérios.

Desse modo, de acordo com a Sociedade Brasileira de Diabetes (2014), devem ser ressaltados alguns aspectos básicos antes de iniciar a terapia medicamentosa em idosos, haja vista que o idoso, frequentemente, é portador de outras doenças, como insuficiência renal, hepática, respiratória, circulatória e cardíaca, às vezes sem expressões clínicas, o que limita a prescrição de antidiabéticos orais e restando a insulina como única opção terapêutica. Assim, são propostas recomendações, com base em níveis de evidência, em que: (A) estudos experimentais e observacionais de melhor consistência; (B) estudos experimentais e 
observacionais de menor consistência; (C) relatos de casos e estudos não controlados; (D) opinião desprovida de avaliação crítica, baseada em consenso, estudos fisiológicos ou modelos animais, conforme ilustrado pela Tabela 1.

Tabela 1: Recomendações e conclusões finais.

\begin{tabular}{|l|c|}
\hline \multicolumn{1}{|c|}{ RECOMENDAÇÃO OU CONCLUSÃO } & GRAU DE RECOMENDAÇÃO \\
\hline O tratamento do idoso diabético deve obedecer aos mesmos princípios dos não idosos. & A \\
\hline $\begin{array}{l}\text { Não há evidências de que o controle glicêmico rigoroso em idosos evita eventos } \\
\text { cardiovasculares. }\end{array}$ & B \\
\hline $\begin{array}{l}\text { A terapia intensificada em idosos diabéticos está associada a um maior risco de } \\
\text { hipoglicemia. }\end{array}$ & B \\
\hline $\begin{array}{l}\text { Não se conseguiu comprovar que a maior mortalidade observada com tratamento intensivo } \\
\text { dos idosos diabéticos se deveria à maior incidência de hipoglicemia. }\end{array}$ & A \\
\hline $\begin{array}{l}\text { Metformina não é contraindicada em idosos, mas quando recomendada, deve-se dar maior } \\
\text { atenção às funções renal, hepática, cardiopulmonar e a quaisquer situações que } \\
\text { predisponham à acidose. }\end{array}$ & B \\
\hline $\begin{array}{l}\text { Insulinoterapia não está contraindicada aos idosos diabéticos, mas esse tipo de tratamento } \\
\text { necessita de cuidados maiores e implica maior risco de hipoglicemia. }\end{array}$ & C \\
\hline $\begin{array}{l}\text { Acarbose pode ser utilizada em idosos diabéticos com mínimo ou nenhum risco de } \\
\text { hipoglicemia, mas a tolerância aos efeitos colaterais é menor. }\end{array}$ & A \\
\hline $\begin{array}{l}\text { Glitazonas podem ser utilizadas em pacientes idosos diabéticos, mas o risco de insuficiência } \\
\text { cardíaca e osteoporose (principalmente em mulheres) limitam o seu uso nessa população. }\end{array}$ & B \\
\hline $\begin{array}{l}\text { Análogos de peptídeo semelhante ao glucagon (GLP-1) e inibidores de dideptidil-IV (DDP- } \\
\text { IV) podem ser usados em idosos diabéticos, com especial atenção à função renal. }\end{array}$ & \\
\hline
\end{tabular}

Fonte: Sociedade Brasileira de Diabetes (2014).

Sob essa perspectiva, o presente estudo tem como objetivo apresentar um relato de caso clínico de episódio de hipoglicemia associado a um distúrbio hipocalêmico em paciente idosa portadora de LADA e, a partir disso, discorrer, sob âmbito fisiológico, esses eventos.

\section{Metodologia}

Este artigo trata-se de um estudo de caso clínico com perspectiva qualitativa e descritiva, que consiste em uma pesquisa em que, em geral, ocorre com coleta direta de dados, cujo o pesquisador é o instrumento indispensável. O presente estudo contou com o aporte de trabalhos acadêmicos entre os anos de 2001 e 2020. Por meio do acesso às principais bases de dados, a saber: dados National Library of Medicine (PubMed MEDLINE), Scientific Electronic Library Online (Scielo), Cochrane Database of Systematic Reviews (CDSR), Google Scholar, Biblioteca Virtual em Saúde (BVS) e EBSCO Information Services, no período compreendido entre março e julho de 2021. O Termo de Consentimento Livre e Assistido (TCLE) foi assinado pelo paciente para coleta de dados neste estudo.

\section{Resultados e Discussão}

Trata-se de paciente do sexo feminino, 65 anos de idade e diagnóstico de diabetes autoimune latente (LADA) em abril de 2019, que se justificou por episódios de hiperglicemias e correlação empírica com doença autoimune já existente na família, posteriormente à dosagem de anticorpos GADA contra as células beta pancreáticas, com marcação de 2000,0 UI/mL (VR: inferior a 10,0 UI/mL), bem como dosagem de hemoglobina glicada, que constou 9,3\%. Dada a identificação, a patologia seguiu-se com controle por meio de modificações dietéticas, administração insulínica de ação intermediária e lenta, a Lantus, e de ação rápida, a Asparte Fiasp, cuja dosagens variam de acordo com refeições, bem como uso diário de 
empagliflozina/linagliptina, ou seja, o medicamento Glyxambi, a fim de melhor controle glicêmico e melhora da qualidade de vida.

A paciente foi admitida no dia 07/09/2020 no pronto atendimento do hospital com quadro de hipoglicemia severa (glicemia capilar de $51 \mathrm{mg} / \mathrm{dl}$ ), rebaixamento do estado de consciência (Glasgow 4 em 15), taquicardia, fraqueza e espasmos musculares, bem como sensação de câimbra nos membros inferiores. Exames laboratoriais mostraram, por meio do método de eletrodo seletivo, que os níveis séricos de potássio estavam em 2,80 mEq/L (VR: 3,50 e 5,50 mEq/L). Foi realizado tratamento com soro glicosado a 50\% e injeção intravenosa de íons potássio, com monitoramento contínuo. Após três horas, os níveis de potássio sérico se normalizaram, repetido o exame de sangue $(3,62 \mathrm{mEq} / \mathrm{L}$, dentro dos mesmos padrões de referência). Após melhora, a paciente apresentou condições de alta hospitalar e seguimento ambulatorial. Relata redução da acuidade visual e realizou consulta com oftalmologista recentemente. Foi feito diagnóstico de catarata bilateral com indicação cirúrgica. Apresenta sinais de neuropatia diabética em membros inferiores com comprometimento diário das atividades, com relatos de formigamento nos pés e sensação de ardência. Retornou dia 05/03/2021 para avaliação de exames laboratoriais, os quais apresentaram descompensação glicêmica, com crises hipoglicêmicas recorrentes durante a noite, com função renal, hepática e cardíaca preservada, conforme demonstrado em exames (Tabela 2).

Tabela 2: Resultados de exames realizados no dia 27/02/2021.

\begin{tabular}{|c|c|c|}
\hline Exames & Valor dosado & Valores de referência \\
\hline Creatinina & $0,87 \mathrm{mg} / \mathrm{dL}$ & Até $1,20 \mathrm{mg} / \mathrm{dL}$ \\
\hline Billirubina total & $0,77 \mathrm{mg} / \mathrm{dL}$ & Até $1,20 \mathrm{mg} / \mathrm{dL}$ \\
\hline Albumina & $4,17 \mathrm{~g} / \mathrm{dL}$ & De 3,4 a $5,2 \mathrm{~g} / \mathrm{dL}$ \\
\hline
\end{tabular}

Fonte: Autores (2021).

Eletrocardiograma e ecocardiograma realizados no dia 25/02//2021 conclusivos para ritmo sinusal regular, sem alterações isquêmicas, função preservada do ventrículo esquerdo, regurgitação aórtica e tricúspide discreta.

\section{Discussão}

O mecanismo de ação da insulina inicia-se pela sua ligação ao receptor de membrana plasmática, que ocorre com alta especificidade e afinidade, provocando mudanças conformacionais que desencadeiam reações modificadoras do metabolismo da célula-alvo, constituindo assim uma resposta celular. Os receptores não são componentes fixos, podendo variar o número de receptores para cada tipo de célula, com isso variando o grau de resposta. A ligação do complexo hormônio-receptor é forte, mas não covalente, sendo equivalente à união de um efetor alostérico com a enzima que o regula (Martins, 2016).

O processo de secreção de insulina estimulada por glicose inicia-se pela difusão facilitada dessa hexose, via GLUT1 ou GLUT2, através do plasmalema da célula beta pancreática, à qual se segue a fosforilação catalisada pela hexoquinase e a metabolização na via glicolítica e no Ciclo de Krebs. O aumento da razão citosólica ATP/ADP, atuando via subunidade receptora de sulfonilureias (SUR1) gera o fechamento de canais $\mathrm{K}+\mathrm{ATP}$, o que resulta na diminuição da saída de $\mathrm{K}+$. O consequente acúmulo desse íon no citosol provoca a despolarização da membrana plasmática e a abertura dos canais de Ca2+ dependentes de voltagem. $\mathrm{O}$ aumento da entrada de $\mathrm{Ca} 2+$ eleva a atividade citosólica deste cátion e desencadeia exocitose dos grânulos de insulina (Haber et al. 2001).

Canais de potássio constituem o mais variado grupo de canais iônicos, consequência da multiplicidade de funções que devem executar. Estas incluem o controle do potencial de repouso da membrana plasmática e a regulação dos potenciais de ação, quanto a seu início, término e periodicidade. São, também, alvo de numerosos hormônios e neurotransmissores, hábeis 
em promover ou inibir a secreção por mecanismos que dependem, em parte, de alterações do potencial da membrana celular (Guyton, 2002).

Existem pelo menos cinco diferentes tipos de canais seletivos de $\mathrm{K}+$ na célula beta, a saber: o canal $\mathrm{K}+$ regulado por ATP; o canal de $\mathrm{K}+$ ativado por $\mathrm{Ca} 2+$; o canal retificador tardio; o canal de $\mathrm{K}+$ ativado por transmissores inibitórios e o canal transiente. Além disso, o potássio também pode fluir através do canal não-seletivo de cátions. Em geral, predomina-se a resposta dos canais K+ATP às concentrações intracelulares de ATP, mas muitos outros nucleotídeos, como o ADP, têm grande influência sobre a frequência de abertura e fechamento desses canais. Assim, considera-se a relação ATP/ADP como o maior determinante da sua atividade (Guyton, 2002).

Em concentrações subestimulatórias de glicose, o potencial de repouso da célula beta é estabelecido pela atividade dos canais $\mathrm{K}+\mathrm{ATP}$ e muitos canais abertos podem ser registrados em células beta desprovidas de suprimento energético. $\mathrm{O}$ fechamento de canais K+ATP pelo ATP representa um mecanismo de ligação entre o metabolismo e os fluxos iônicos. A glicose é capaz de inibir a atividade de canais K+ATP. É improvável, contudo, que a extensão da inibição do efluxo de K+ por ação em canais K+ATP determine sozinha o montante da insulina secretada. Desse modo, a inibição do efluxo de K+, pelo metabolismo de nutrientes, seria apenas uma parte do processo, e efeitos adicionais do metabolismo participariam da cascata de fluxos iônicos (Lima, 2018).

Assim, a ativação do receptor gera um sinal que, eventualmente, resulta na ação da insulina sobre a glicose, lipídeos, o metabolismo de proteínas, garantindo diferentes efeitos metabólicos. Os efeitos promotores do crescimento de insulina aparentemente ocorrem através da ativação de receptores da família de fatores de crescimento semelhantes à insulina. Anormalidades no número de receptores de insulina, falha na atividade quinase do receptor e os vários passos de sinalização pós-receptor na ação da insulina ocorrem em estados de doença que conduzem a resistência dos tecidos (Martins, 2016).

Visto isso, ao se considerar as alterações de potencial de repouso da membrana neuronal ocorrem em decorrência da abertura ou fechamento de canais de potássio sensíveis a ATP (K+ ATP) em mecanismo semelhante ao que ocorre nas células $\beta$-pancreáticas, onde o papel desse canal está bem definido. Além disso, já se sabe da importância desses K + ATP em neurônios, onde participam da manutenção do potencial de repouso da membrana e na analgesia periférica induzida por opioides (Lima, 2018). Portanto, glicose e insulina poderiam atuar por meio dos K + ATP, sendo que a hiperglicemia poderia induzir uma despolarização enquanto que a insulina induziria uma hiperpolarização da membrana neuronal, utilizando esses canais. Sendo assim, os canais de potássio sensíveis ao ATP nos neurônios sensoriais podem ser importantes para o desenvolvimento da neuropatia diabética e possivelmente um alvo terapêutico para a prevenção ou tratamento desta complicação crônica associada ao diabetes (Lima, 2018).

Como o potássio é um eletrólito de suma importância para a manutenção do potencial de membrana das células musculares e nervosas, sendo o cátion mais abundante no meio intracelular, faz parte do funcionamento adequado dos músculos, inclusive o cardíaco. A hipocalemia pode ocorrer devido à perda excessiva do íon pelo trato gastrintestinal secundariamente a vômitos, diarreia ou uso excessivo de laxantes, bem por aumento dos níveis insulínicos. Certos medicamentos, como a insulina sintética, fazem com que mais potássio se mova do sangue para as células, ou seja, do meio extracelular para o intracelular, podendo resultar em hipocalemia temporária.

A ação da insulina, endógena ou exógena, é, principalmente, no músculo esquelético, com aumento da atividade da bomba de sódio, potássio e ATPase (Na/K/ATPase), com aumento da entrada de potássio no meio intracelular, diminuindo os níveis séricos desse íon. Assim, o aumento da insulina predispõe a hipocalemia, e isto é particularmente observado em pacientes na fase de recuperação de cetoacidose diabética, bem como em quadros de hipoglicemias críticas, como no caso apresentado (Burton, 2001). A hipoglicemia, que pode ser o resultado da administração excessiva de insulina durante o tratamento inicial ou da manutenção da infusão de insulina sem a reposição concomitante de glicose, seja via dietética, seja via 
soluto, pode ocasionar, conforme ilustrado pelo caso exposto, a hipocalemia severa após insulinoterapia, que irá deslocar o potássio do meio extracelular para o intracelular, levando em consideração que a insulina é um dos mecanismos mais poderosos para deslocamento do potássio para o interior da célula (Burton, 2001).

Pequenas alterações no potássio sérico podem implicar profunda alteração na condução e excitabilidade do coração, podendo alterar a função e o ritmo cardíacos e, inclusive, causar parada cardiorrespiratória. Casos graves de hipocalemia, aqueles que podem surgir se o nível de potássio no sangue for $(<2,5 \mathrm{mEq} / \mathrm{L})$ podem resultar em paresias e batimento cardíaco irregular, colocando a vida do paciente em perigo, bem como lesões cerebrais e de outros órgãos podem ocorrer devido a hipoxia resultante de parada cardíaca ou respiratória secundária à hipocalemia e insuficiência renal aguda pode estar associada com rabdomiólise secundária à hipocalemia (Neto, 2011).

Em pacientes que apresentem exame admissional com hipocalemia grave $(<3,3 \mathrm{mEq} / \mathrm{l})$, pode-se iniciar a reposição de potássio juntamente com a hidratação venosa, postergando o início da insulinização. Tal conduta se baseia no fato de que graus tão severos de hipocalemia oferecem risco de complicações, como arritmias cardíacas, parada cardiorrespiratória ou ainda de fraqueza da musculatura respiratória, com potencial evolução para insuficiência respiratória aguda (Barone, 2017). Desse modo, é notório que as crises de hipoglicemia são fatores complicadores no tratamento do diabetes, ao induzir, de forma deficiente, mecanismos de contra-regulação. À medida que a glicemia cai, sinais clínicos de disfunção cerebral vão aparecendo, acompanhados por profundas alterações nas sinapses cerebrais e na neurotransmissão. Aliado a isso, a queda sérica de potássio apresenta-se como consequências sérias deste desequilíbrio, sendo, por isso, de suma importância a investigação de efeitos deletérios decorrentes destes episódios, como a mialgia, fraqueza muscular, rabdomiólise, poliúria, íleo paralítico (decorrente das alterações de repolarização muscular), intolerância a glicose, alcalose metabólica e distúrbios do ritmo cardíaco (Nery, 2008).

\section{Conclusão}

Portanto, é importante ressaltar que durante o prognóstico de indivíduos com diabetes mellitus e com doença cardiovascular prévia, o tratamento intensivo objetivando glicemias próximas da normalidade pode ser instituído, mas com cautela para que não ocorram hipoglicemias graves. Ademais, pode-se concluir que a hipoglicemia aguda resulta em complexos e ainda mal compreendidos fenômenos. Assim, para prática diária e para o melhor conhecimento da associação de hipoglicemias e seus mecanismos fisiopatológicos com a mortalidade cardiovascular há necessidade de mais estudos com desenhos apropriados.

Haja vista que a presença de níveis séricos baixos de potássio podem ter muitas causas, como vômitos, diarreia, distúrbios da glândula adrenal, uso de diuréticos, albuterol e insulina, a sintomatologia do paciente em estado hipoglicêmico é primordial para a suspeita de hipocalemia, sendo os achados mais comuns a câimbra e fraqueza muscular, o que justifica a medição dos níveis de potássio no sangue, e se confirmado, solicitação de eletrocardiograma a fim de investigar a presença de ritmos cardíacos anormais. Como os suplementos tomados via oral são ineficazes em uma situação de hipocalemia, o potássio deve ser reposto e administrado via intravenosa. Diante do caso exposto, o distúrbio hipocalêmico provavelmente foi decorrente do estado de hipoglicemia severa, causado pelo aumento dos níveis de insulina e, consequentemente, pelo deslocamento de potássio para os compartimentos intracelulares, diminuindo, assim, as concentrações plasmáticas desse eletrólito. 


\section{Referências}

Azevedo, L. C. P., Taniguchi, L. U., Ladeira, J. P. \& Besen, B. A. M. P. (2013). Medicina Intensiva: abordagem prática, Manole, 535 - 555.

Barone, B., Rodacki, M., Cenci, M. C. P., Zajdenverg, L., Milech, A. \& Oliveira, J. E. P. (2007). Cetoacidose diabética em adultos: atualização de uma complicação antiga. Arq. Bras. Encodrinol. Metab., 51 (9), 1434-1447.

Burton, R. (2001). Hypokalemia. In: Clinical physiology of acid-base and electrolyte disorders. (5a ed.), McGraw-Hill, 836-885.

Buzzetti, R., Tuomi, T., Mauricio, D., Pietropaolo, M., Zhou, Z. \& Leslie, R. D. (2020). Management of Latent Autoimmune Diabetes in Adults: A Consensus Statement From an International Expert Panel. Diabetes, 69 (10), 2037-2047.

Calsolari, M. R., Rosário, P. W. S., Reis, J. S., Silva, S. C. \& Purisch, S. (2008). Diabetes autoimune latente do adulto ou diabetes melito tipo 2 magro? Arq. Bras. Encodrinol. Metab., 52 (2), 1-6.

Couri, C. E., Oliveira, M. C. B., Stracieri, A. B. P. L., Moraes, D. A. \& Voltarelli, J. C. (2009). C-peptide levels and insulin independence following autologous nonmyeloablative hematopoietic stem cell transplantation in newly diagnosed type 1 diabetes mellitus. JAMA, 301 (15), $1573-1579$.

Groop. L. C., Bottazzo, G. F. \& Doniach, D. (1986). Islet cell antibodies identify latent type I diabetes in patients aged $35-75$ years at diagnosis. Diabetes, 35 (2), 237-241.

Guyton, A. C. (2017). Tratado de Fisiologia Médica. Editora Elsevier. (13a ed.).

Haber, E. P., Curi, R., Carvalho, C. R. O. \& Carpinelli, A. R. (2001). Secreção da insulina: efeito autócrino da insulina e modulação por ácidos graxos. Arq. Bras. Encodrinol. Metab., 45 (3), 1-9.

Jones, A. G., McDonald, T. J., Shields, B. M., Hagopian, W. \& Hattersley, A. T. (2021). Latent Autoimmune Diabetes of Adults (LADA) is likely to representant a mixed population of autoimmune (type 1) and nonautoimmune (type 2) diabetes. Diabetes Care, 44 (1), 1-9.

Lima, T. C. (2018). Hiperglicemia induz hiperalgesia mecânica e despolarização do potencial de repouso da membrana de neurônios nociceptivos primários: papel dos canais de potássio sensíveis ao ATP. Universidade Federal de Uberlândia. 69 p.

Martins, F. S. M. (2016). Mecanismos de ação da insulina. Universidade Federal do Rio Grande do Sul, 1-13.

Nery, M. (2008). Hipoglicemia como fator complicador no tratamento do diabetes melito tipo 1. Arq. Bras. Encodrinol. Metab., 52 (2), 1-11.

Neto, R. A. B. (2011). Hipocalemia. MedicinaNet, https://www.medicinanet.com.br/conteudos/casos/4130/hipocalemia.htm\#: :text=Esse\%20gradiente\%20e ntre $\% 20$ os\%20dois,e \%20inclusive \%20causar\%20parada\%20cardiorrespirat\%C3\%B3ria.

Pieralice, S. \& Pozzilli, P. (2018). Latent Autoimmune Diabetes in Adults: A Review on Clinical Implications and Management. Diabetes \& Metabolism Journal, 42 (6), 451-464,

Pozzilli, P. \& Mario, U. D. (2001). Autoimmune diabetes not requiring insulin at diagnosis (latent autoimmune diabetes of the adult): definition, characterization, and potential prevention. Diabetes Care, 24 (8), 1460-1467.

Rastergar, A. \& Soleimani, M. (2001). Hypokalemia and hyperkalaemia. Postgraduate Medical Journal, 77 (914), $159-764$.

Rosário, P. W. S., Reis, J. S., Amim, R., Fagundes, T. A., Calsolari, M. R., Silva, S. C. \& Purisch, S. (2005). Comparison of clinical and laboratory characteristics between adult-onset type 1 diabetes and latent autoimmune diabetes in adults. Diabetes Care, 28 (7), $1803-1804$.

Silva, M. E. R. (2007). Precisamos diagnosticar o diabetes Latente Autoimune do Adulto (LADA)? Arq. Bras. Encodrinol. Metab., 51 (1).

Singer, G. G. \& Brenner, B. M. (2008). Distúrbios hidroeletrolíticos. In: FALCI, et al. Harrison Medicina Interna, (17a ed.), McGraw-Hill Interamericana do Brasil.

Sociedade Brasileira de Diabetes (SBD) (2020). Diretrizes da Sociedade Brasileira de Diabetes: 2020-2021. Clannad.

Sociedade Brasileira de Diabetes (SBD) (2014). Tratamento de pacientes idosos com diabetes: 2014-2015. Clannad. 